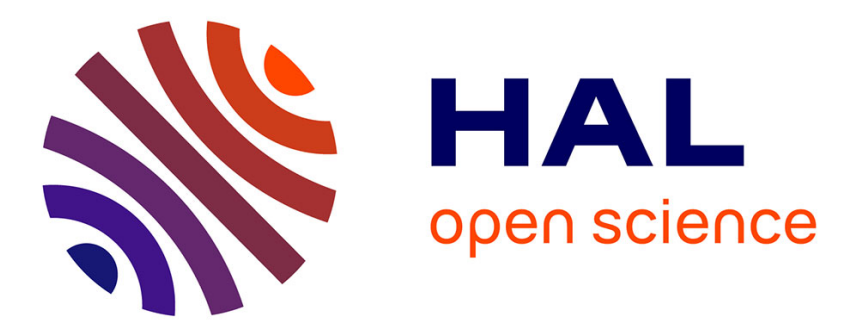

\title{
Magnetorheology of fiber suspensions. I. Experimental
}

Modesto Lopez-Lopez, Pavel Kuzhir, Georges Bossis

\section{To cite this version:}

Modesto Lopez-Lopez, Pavel Kuzhir, Georges Bossis. Magnetorheology of fiber suspensions. I. Experimental. Journal of Rheology, 2009, 53 (1), pp.115-126. 10.1122/1.3005402 . hal-00441005

\section{HAL Id: hal-00441005 \\ https://hal.science/hal-00441005}

Submitted on 10 Feb 2010

HAL is a multi-disciplinary open access archive for the deposit and dissemination of scientific research documents, whether they are published or not. The documents may come from teaching and research institutions in France or abroad, or from public or private research centers.
L'archive ouverte pluridisciplinaire HAL, est destinée au dépôt et à la diffusion de documents scientifiques de niveau recherche, publiés ou non, émanant des établissements d'enseignement et de recherche français ou étrangers, des laboratoires publics ou privés. 


\section{TITLE OF THE ARTICLE:}

\section{Magnetorheology of fiber suspensions. I. Experimental}

\section{AUTHORS:}

Modesto T. López-López, ${ }^{1}$ Pavel Kuzhir, Georges Bossis

Laboratoire de Physique de la Matière Condensée, CNRS UMR 6622 Université de Nice, Parc Valrose, 06108 Nice Cedex 2, France

${ }^{1}$ Present address: Department of Applied Physics, University of Granada, Avda. Fuentenueva s/n, 18017 Granada, Spain.

\section{CORRESPONDING AUTHOR:}

Modesto T. López-López. E-mail: modesto@ugr.es

\section{SYNOPSIS}

This work reports a detailed study on the shear magnetorheology of suspensions of magnetic microfibers. The steady-state regime was investigated using a controlled-stress rheometer, for different concentrations of particles and under the presence of a broad range of applied magnetic fields (up to $512 \mathrm{kA} \mathrm{m}^{-1}$ ). The results were compared with those obtained for conventional magnetorheological fluids (suspensions of magnetic microspheres). It was found that the suspensions of magnetic fibers show an enhanced magnetorheological effect. We proposed the existence of field-dependent solid friction between fibers as the main physical reason for this enhancement. In order to ascertain the relevance of the interfiber solid friction, the microscopic structure of fiber suspensions was investigated using an optical microscope. In the absence of applied field, fibers form an entangled network with approximately isotropic orientation. Upon magnetic field application, the fiber network becomes deformed and 
approximately aligned with the field direction. Nonetheless, interfiber solid friction hinders a complete alignment of the fibers with the field, and the fiber network remains entangled.

\section{INTRODUCTION}

Magnetorheological (MR) suspensions are complex fluids that undergo a tunable and reversible transition from a solid-like to a liquid-like state upon magnetic field application [Bossis et al. (2002); Ginder (1998); Rankin et al. (1998); See (2003)]. This property is the base of many applications of MR suspensions in medicine [Liu et al. (2001); Sheng et al. (1999); Wilhelm et al. (2005)] and engineering [Bica (2002); Carlson et al. (1996); Jolly et al. (1999); Kordonski and Golini (2002)]. Besides medical and engineering applications, current research on MR suspensions focuses on: (i) the settling and redispersion of the magnetic particles of MR suspensions [Garay and Bilbao (2007); López-López et al. (2006b); Ngatu and Wereley (2007); Pu et al. (2006)]; and (ii) developing new MR suspensions with enhanced properties. As a result of (ii), new MR suspensions constituted by micron-sized magnetic particles dispersed in ferrofluids [López-López et al (2005, 2006a); Ngatu and Wereley (2007); Viota et al. (2007); Wereley et al. (2006)] or in ionic liquids [GuerreroSanchez et al. (2007)] have recently been prepared and characterized.

Furthermore, in one of our recent works [López-López et al. (2007)] the synthesis of MR suspensions constituted by magnetic cobalt microfibers dispersed in a carrier liquid was reported. In this work, we also reported some preliminary data on the yield stress of a suspension containing 5 vol.\% of cobalt microfibers. We found that this suspension developed much larger values of the yield stress than the equivalent suspension of cobalt microspheres at medium and high magnetic field strength. The shape anisotropy of the fibers and the possible existence of friction between them could be some of the reasons for this enhancement of the MR effect. Besides, an interesting rheological behavior is expected for such MR fiber 
suspensions, combining the behaviors observed in nonmagnetic fiber suspensions and in conventional MR suspensions (suspensions of magnetic microspheres).

We also reported elsewhere an experimental study on the shear and squeeze rheometry of suspensions of magnetic polymerized chains (see the work by Kuzhir et al. (2007)). Independently, Bell et al. (2007a; 2007b; 2008), reported measurements of the yield stress of suspensions of magnetic wires. These authors found an increase of the yield stress of magnetic wire suspensions when compared with that of suspensions of spherical particles. Later, they analyzed the effect of substituting microspheres by nanowires in MR fluids, and no relevant change of the yield stress was found when the relative concentration of wires was increased up to $10 \%$ [Ngatu et al. (2008)]. Unfortunately, the magnetic properties of the particles were not characterized and, as the same authors stated, "it is unclear how the magnetic saturation of the particles quantitatively affects the yield stress of the microwirebased suspensions" [Bell et al. (2008)].

The aim of the current work is to study in detail the MR properties of suspensions of magnetic fibers and to analyze the forces that govern the MR behavior. With this objective, the steady-shear flow of suspensions containing 1, 3, 5 and 7 vol.\% of cobalt fibers or cobalt spheres was studied and the corresponding yield stresses were obtained. The effect on the MR properties of the applied magnetic field was investigated for a wide range of magnetic field strengths (from $6 \mathrm{kA} \mathrm{m}^{-1}$ up to $512 \mathrm{kA} \mathrm{m}^{-1}$ ). In addition, the internal structure in the absence and presence of magnetic field was experimentally analyzed by means of optical microscopy.

\section{EXPERIMENTAL METHODS}

In this work, two kinds of MR suspensions were prepared: suspensions of cobalt microfibers and suspensions of cobalt microspheres. Both cobalt fibers and cobalt spheres 
were prepared by reduction of cobalt ions in polyols as described in [López-López et al. (2007)]. Anisotropic growth in the synthesis of cobalt fibers was induced by means of the application of a magnetic field during the whole synthesis time. Cobalt fibers were polydisperse with average length and width of $60 \pm 24 \mu \mathrm{m}$ and $4.8 \pm 1.9 \mu \mathrm{m}$ respectively, as shown by SEM microscopy [López-López et al. (2007)]. As an example, a SEM picture of the cobalt fibers is shown in Figure 1. Cobalt spheres were also polydisperse with an average diameter of $1.34 \pm 0.40 \mu \mathrm{m}$ [López-López et al. (2007]. The magnetization, $M$, of both the cobalt fibers and the cobalt spheres was measured at $20{ }^{\circ} \mathrm{C}$ as a function of the magnetic field strength, $H$, using a Squid Quantum Design MPMS XL magnetometer. Figure 2 shows the obtained magnetization curves an the fits to the Fröhlich-Kennelly law [Jiles (1991)]:

$$
M=\frac{\chi_{i} M_{S} H}{M_{S}+\chi_{i} H}
$$

where $\chi_{i}$ is the magnetic susceptibility at $H \rightarrow 0$, and $M_{S}$ is the saturation magnetization. As observed in Figure 2, the magnetization curves of cobalt fibers and cobalt spheres are very similar, the remnant magnetization being the only considerable difference: 53 and $5 \mathrm{kA} \mathrm{m}^{-1}$ for cobalt fibers and cobalt spheres respectively. From the fits to the Fröhlich-Kennelly law, a saturation magnetization of $1366 \pm 8,1464 \pm 14 \mathrm{kA} \mathrm{m}^{-1}$ and a initial susceptibility of $17.3 \pm$ $0.6,11.8 \pm 0.5$ are obtained for cobalt fibers and cobalt spheres respectively. Notice that the values of the saturation magnetization obtained by fitting the Fröhlich-Kennelly law are a bit higher than the values obtained by averaging the experimental magnetization data in the high field plateau: $1320,1360 \mathrm{kA} \mathrm{m}^{-1}$ for cobalt fibers and cobalt spheres respectively.

MR suspensions were prepared by dispersing appropriate amounts of cobalt fibers or cobalt spheres in silicone oil (Rhodorsil ${ }^{\mathrm{R}}$; VWR International) with viscosity $0.479 \mathrm{~Pa} \cdot \mathrm{s}$ at 25 ${ }^{\circ} \mathrm{C}$. Aluminum stearate (molecular formula: $\mathrm{Al}\left(\mathrm{C}_{18} \mathrm{H}_{35} \mathrm{O}_{2}\right)_{3}$; technical grade; Sigma-Aldrich) was used as surfactant to avoid particle irreversible aggregation. The concentration of 
aluminum stearate was $0.007 \mathrm{~g}$ per $\mathrm{g}$ of cobalt. Cobalt concentration of the suspensions used in the MR tests was 1, 3, 5 and 7 vol.\%. A detailed description of the procedure followed in order to coat the particles with aluminum stearate and to homogenize the suspensions can be found in [López-López et al. (2006b)].

Magnetorheological properties were measured using a Haake RS150 controlled stress rheometer. The magnetic field was applied in the vertical direction using two separate devices: (i) a coil, which was used for generating fields up to $36 \mathrm{kA} \mathrm{m}^{-1}$; (ii) a noncommercial electromagnet, specially designed for its use in the above-mentioned rheometer (see the scheme in Figure 3). This electromagnet allows reaching fields up to $580 \mathrm{kA} \mathrm{m}^{-1}$ in the measuring gap -the radial distribution of the magnetic field in the measuring gap for a given electric current in the coil is shown in Figure 4. A cone-plate configuration $(35 \mathrm{~mm}$ in diameter and 2 degrees in angle) was used when the magnetic field was generated with the coil, and a plate-plate configuration of diameter $20 \mathrm{~mm}$ and gap $0.300 \mathrm{~mm}$ when it was generated with the electromagnet.

In order to check whether the fibers wore out or fragment under shear, they were observed in the microscope before and after the MR experiments, and no important changes in their integrity were observed. Therefore, it can be concluded that the fibers did not wear out or fragment under shear.

Internal structures in the absence and presence of horizontal and vertical magnetic field were analyzed using an optical microscope with objectives $\times 10, \times 60$ and $\times 150$. A coil was used for generating vertical magnetic fields up to $32.58 \mathrm{kA} \mathrm{m}^{-1}$ of strength. The horizontal field (up to $32 \mathrm{kA} \mathrm{m}^{-1}$ of strength) was applied using an electromagnet. Suspensions used in these experiments were prepared as described above and then diluted down to 0.1 vol.\% of cobalt particles. Right before the observation, suspensions were redispersed by means of mechanical stirring and sonication. Then a drop of suspension was 
placed between two horizontally parallel glass slides. The gap between the glass slides was fixed to $0.15 \mathrm{~mm}$. Observations were conducted upon increasing magnetic field strengths.

\section{MAGNETORHEOLOGY}

In a typical experiment, suspensions were initially pre-sheared at a large shear rate $\left(\sim 400 \mathrm{~s}^{-1}\right)$ to ensure reproducible initial conditions. Afterwards, suspensions were subjected to shear stress ramps (in the absence and presence of magnetic field), with a 10-s interval between each stress value, and the corresponding shear rates and dynamic viscosities were measured. For each sample and each value of the magnetic field strength, data acquisition was performed in linear and in logarithmic modes. In both cases the shear stress ramp was adjusted for each applied magnetic field, in such a way that at least 50 different measuring points were obtained in the shear rate interval $0-500 \mathrm{~s}^{-1}$. In this way, we were able to determine with precision both the static and the dynamic yield stresses. In all cases we checked that the curves obtained in the linear mode approximately collapsed with the curves obtained in the logarithmic mode. As an example, the rheograms (shear stress vs. shear rate) of the suspension containing $5 \mathrm{vol} \%$ of cobalt fibers for some values of the magnetic field strength are shown in Figure 5. Note the tunable plastic behavior conferred by the applied magnetic field, which is characteristic of MR fluids.

Let us now focus on the comparison of the MR behavior of suspensions of cobalt fibers and suspensions of cobalt spheres. The shear stress of suspensions containing 3 vol.\% of cobalt spheres or fibers, subjected to a magnetic field strength of $30.55 \mathrm{kA} \mathrm{m}^{-1}$, is plotted in Figure 6 as a function of the shear rate -similar trends are obtained for other values of the magnetic field and/or particle concentration. From curves like those shown in Figure 6a 
(linear data acquisition) the values of the dynamic (Bingham) yield stress were obtained by fitting the Bingham equation [Larson (1999)]:

$$
\tau=\tau_{y B}+\eta_{p l} \dot{\gamma}
$$

where $\tau$ is the shear stress, $\tau_{y B}$ the dynamic (Bingham) yield stress and $\eta_{p l}$ the plastic viscosity. It is worthy to note that the Bingham yield stress represents the stress needed to continuously break the aggregates that reform in the presence of the magnetic field [Volkova et al. (2000)]. Alternatively, the MR effect of MR fluids can be characterized by the values of the static (frictional) yield stress. This one represents the stress for which the field-induced structures either break in the middle or slip on the moving cone or plate of the rheometer [Volkova et al. (2000)]. Its value can be evaluated from curves like those in Figure $6 \mathrm{~b}$ by extrapolating the stress of the pseudoplateau region to zero-shear rate. Before analyzing in detail the values of the yield stress, notice that a similar trend is obtained for both cobalt fibers and cobalt spheres, but with shear stresses that, for the same shear rate, are always higher for the suspension of cobalt fibers than for the suspension of cobalt spheres (see Figure 6). In other words, in the presence of field, suspensions of cobalt fibers exhibit higher values of the yield stress and plastic viscosity than suspensions of cobalt spheres.

In order to analyze in detail the MR effect developed by suspensions of cobalt spheres and fibers, we plotted the values of the static (Figure 7) and dynamic (Figure 8) yield stress as a function of the magnetic strength. As observed in Figures 7 and 8, the field-induced static and dynamic yield stresses increase with both solid volume fraction, $\phi$, and magnetic field strength, as expected for MR fluids. From data on Figure 8, mean dependencies of the dynamic yield stress on $\phi^{(1.5 \pm 0.6)}$ and $\phi^{(1.1 \pm 0.3)}$ are obtained for fiber suspensions and spheres suspensions respectively. In all cases, the increase of the yield stress with magnetic field strength is very sharp at low field and then, it tends to saturation as the field approaches to the value required to magnetically saturate the cobalt particles. Notice also, that the values of the 
dynamic yield stress are always higher than the corresponding values of the static yield stress. More interesting is the fact that the values of both the static and the dynamic yield stress for suspensions of cobalt fibers are approximately 2-3 times higher than the corresponding (equal solid concentration and magnetic field) values for suspensions of cobalt spheres. In our opinion, this is a very interesting result from both a fundamental and a technological point of view. The physical reason for this enhancement of the MR effect when magnetic fibers are used for preparing MR fluids, likely has its origin in the solid friction between fibers. Note that no effect of particle size on the MR properties of these MR fluids is expected, since it has been shown that this effect is negligible for particle sizes larger than $100 \mathrm{~nm}$ [Bossis et al. (2008)]. In addition, theoretical estimations show that the van der Waals interaction is about five orders of magnitude weaker than the magnetic interaction (see companion paper).

In suspensions of magnetic spherical particles, friction is negligible in comparison with the other forces (dipole-dipole magnetic interaction and hydrodynamic interaction). On the contrary, in suspensions of magnetic fibers, solid friction between fibers plays an important role. In the absence of field, fibers are structured in randomly oriented networks. Under this condition, when fiber suspensions are subjected to a shear stress, flowing is hindered by the solid friction between fibers and a nonzero yield stress is measured at zero field. Under magnetic field application, fibers experience attractive magnetic forces and, therefore, the solid friction between fibers, which is proportional to the normal contact force between fibers, increases. This increase in solid friction involves an increase of the resistance of the suspensions to flow. As a consequence, the resulting field-induced yield stress of a fiber suspension must be promoted by the combined effect of friction and magnetic attraction. In the next section some microscopic observations that sustain these arguments are shown.

\section{IV.STRUCTURES IN SUSPENSIONS OF MAGNETIC FIBERS}


Some photos of planar structures of diluted suspensions of cobalt fibers (solid concentration 0.1 vol.\%) confined between two parallel glass slides (the gap was fixed to 0.15 $\mathrm{mm}$ ) are shown in Figure 9(a-e). As seen in Figure 9a, in the absence of magnetic field the fibers form an entangled network with approximately isotropic orientation of fibers, and even at low fiber concentration ( $0.1 \mathrm{vol} . \%)$, each fiber seems to have at least a few contact points with the neighboring ones. It can also be observed that individual fibers are gathered together in aggregates, even though, as mentioned in section II, the suspensions were carefully dispersed right before the observations. Such aggregation in the absence of magnetic field could be due to the combination of different effects: 1) magnetic attraction between fibers because of their remnant magnetization; 2) short range attractive forces: van-der-Waals or electrostatic; and 3) mechanical cohesion between rough fiber surfaces. Such cohesion is likely due to the solid friction between fibers and could involve an important contribution to the flocculation of the fiber suspension. Note that the flocculation of pulp suspensions is a well-known phenomenon and was first reported by Mason (1950). Later, Schmid et al. (2000) and Switzer and Klingenberg (2004) performed particle-level simulations of such suspensions and attributed the fiber flocculation to interfiber friction.

When a magnetic field parallel to the glass slides is applied, the fiber network becomes deformed and approximately aligns with the field direction (see Figure 9b). Notice that the fiber network remains entangled, the fibers linked (chained) to the neighboring ones and, therefore, there is not a complete alignment with the field. This can be explained by the solid friction between fibers that hinders their motion and does not allow them to get completely aligned with the field. Hence, the structure observed is not at equilibrium. Otherwise, without friction, the free energy of the fiber suspension would have been minimized, and a structure with all the fibers aligned completely with the magnetic field, joined end by end with the 
neighboring ones, would have been observed [Bossis et al. (2002)]. A zoomed view of the fiber network upon magnetic field application is presented in Figure 9c. As observed, the fibers are rather polydisperse and have an irregular rough surface. They are linked to each other either by their extremities or by their lateral sides. In the latter situation, two contacting fibers either are attached by their lateral sides or cross each other at some angle. It seems that any type of interfiber contact is equiprobable.

Alternatively, when a magnetic field normal to the glass slides is applied, the fibers tend to become aligned in the vertical plane, i.e. transversely to the glass slides (Figure 9d). However, as can be observed, some fiber aggregates are so big that they cannot be aligned in the vertical plane because their movement is restricted by the gap between the glass slides. And even smaller fiber aggregates do not get strictly perpendicular to the glass slides -fibers are always attached to the neighboring ones by magnetic and friction forces. Note that this structure is rather different from the column-like structure observed in suspensions of spherical magnetic particles (see Figure 4a in Bossis et al. (2002)). Notice also that when this fiber suspension is sheared (the upper glass slide is displaced horizontally), under the presence of vertical magnetic field, the fiber aggregates get more oriented in the direction of shear (see Figure 9e). Thus, we believe that upon magnetic field application, the fibers gather into aggregates, which span the gap between the glass slides, and they are leant, when sheared, in the direction of the shear.

Finally, a photo of a 3D structure of a model fiber suspension consisting of steel rods (15 $\mathrm{mm}$ in length and $1 \mathrm{~mm}$ in diameter) in silicone oil, under the presence of applied magnetic field, is shown in Figure 9f. Similarly to the planar structures discussed above, the fibers form a dendrite-like structure oriented preferably along the magnetic field lines. To model the rheology of magnetic fiber suspensions, a precise knowledge of the types of interfiber contacts that are favored is desired. As seen in Figure 9f (and other pictures for 
cobalt fibers, not shown here for brevity), most of the contacts between fibers are either sideby-side or side-by-end, while end-by-end are infrequent. In fact, this model structure shown in Figure 9f is quite similar to the structure shown in Figure 9c. In both cases the fibers can either attach to neighboring ones by their lateral side (line contact) or cross each other at a certain angle (point contact).

The existence of different types of interfiber contacts is an essential point that must be taken into account to theoretically model the magnetorheology of suspensions of magnetic fibers. This is done in the companion paper, where we introduce some microstructural models for magnetic fiber suspensions and explain the enhanced MR response of these suspensions in terms of interfiber solid friction. Our theory covers the elastic regime of the shear deformation (before the flow onset) and combines the features of the point-wise interaction theory developed by Toll and Manson (1994) for classical fiber suspensions and the features of the column structure and zigzag structure models for classical MR suspensions [Bossis et al. (1997); Volkova (1998)].

\section{CONCLUSIONS}

In this work, the shear rheology of suspensions of magnetic fibers has been studied in detail. The effect of both magnetic field intensity and fiber volume fraction on the rheological properties has been analyzed. It has been found that magnetic fiber suspensions behave as MR fluids -i.e. upon magnetic field application they develop tunable yield stress. The increase of the yield stress with magnetic field strength is very sharp at low field. At high field it tends to saturation, as the magnetization of the cobalt fibers is progressively saturated. The fieldinduced yield stress has also been found to increase faster than linearly with the concentration of fibers in suspension. 
Furthermore, the values of the yield stress obtained for suspensions of cobalt fibers have been compared with those obtained for suspensions of cobalt spheres. It has been found that for the same particle concentration and applied magnetic field strength, suspensions of cobalt fibers develop yield stresses 2-3 times higher than suspensions of cobalt spheres. The existence of field-dependent solid friction between fibers is likely the physical reason for this enhancement.

Finally, the microscopic structures developed in suspensions of magnetic fibers have been explored. In the absence of magnetic field, fibers form an entangled network with approximately isotropic orientation. Upon magnetic field application, the fiber network tends to become aligned with the field. However, friction between fibers hinders their motion and does not allow a complete alignment with the field. In this "equilibrium" state, all kinds of contacts between fibers, side-by-side, side-by-end, or end-by-end, are possible, the third one being the less frequent.

\section{ACKNOWLEDGEMENTS}

We would like to thank undergraduate student Trystan Domenech for helping with experiments. Eureka E! 3733 Hydrosmart project and "Conseil Régional PACA" (Biomag project) are acknowledged for their financial support. One of the authors (M.T.L.-L.) also acknowledges financial support by Secretaría de Estado de Universidades e Investigatigación (Postdoctoral Fellowship Program, MEC, Spain) and by the University of Granada (Spain).

\section{REFERENCES}

Bell, R. C., E. D. Miller, J. O. Karli, A. N. Vavreck and D. T. Zimmerman, in "Electrorheological Fluids and Magnetorheological Suspensions, Proceedings of the $10^{\text {th }}$ 
International Conference on ERMR 2006," Influence of particle shape on the properties of magnetorheological fluids. F. Gordaninejad, O. A. Graeve, A. Fuchs and D. York, eds., World Scientific Publishing Company, Inc., New Jersey, 2007a.

Bell, R. C., E. D. Miller, J. O. Karli, A. N. Vavreck and D. T. Zimmerman, "Influence of particle shape on the properties of magnetorheological fluids," Int. J. Mod. Phys. B 21, 50185025 (2007b).

Bell, R. C., J. O. Karli, A. N. Vavreck, D. T. Zimmerman, G. T. Ngatu and N. M. Wereley, "Magnetorheology of submicron diameter iron microwires dispersed in silicone oil," Smart Mater. Struct. 17, 015028 (2008).

Bica, I., "Damper with magnetorheological suspension,” J. Magn. Magn. Mater. 241, 196-200 (2002).

Bossis, G., N. Kchit, P. Kuzhir, M. T. López-López, A. Meunier, in " $11^{\text {th }}$ International Conference on Electrorheological Fluids and Magnetorheological Suspensions, Book of Abstracts," Role of particle size and interparticle gap on the yield stress and conductivity of MR suspensions. S. Odenbach and D. Borin, eds., Technische Universität Dresden, Dresden, 2008.

Bossis, G., E. Lemaire, O. Volkova and H. Clercx, "Yield stress in magnetorheological and electrorheological fluids: A comparison between microscopic and macroscopic structural models,” J. Rheol. 41, 687-704 (1997). 
Bossis, G., O. Volkova, S. Lacis and A. Meunier, in "Ferrofluids," Magnetorheology: Fluids, Structures and Rheology. S. Odenbach, ed., Springer, Berlin, 2002.

Carlson, J. D., D. M. Catanzarite and K. A. StClair, "Commercial magneto-rheological fluid devices,” Int. J. Mod. Phys. B 10, 2857-2865 (1996).

Garay, D. and P. Bilbao, "Magnetorheological fluids with enhanced antisettling behaviour," Sens. Lett. 5, 81-85 (2007).

Ginder, J. M., “Behavior of magnetorheological fluids,” MRS Bull (Aug) 26-29 (1998).

Guerrero-Sanchez, C., T. Lara-Ceniceros, E. Jimenez-Regalado, M. Rasa and U. S. Schubert, “Magnetorheological fluids based on ionic liquids,” Adv. Mater. 19, 1740-1747 (2007).

Jiles, D., Introduction to Magnetism and Magnetic Materials. Chapman \& Hill, London, 1991.

Jolly, M. R., J. W. Bender, J. D. Carlson, "Properties and applications of commercial magnetorheological fluids,” J. Intell. Mater. Syst. Struct. 10, 5-13 (1999).

Kordonski, W. and D. Golini, "Multiple application of magnetorheological effect in high precision finishing,” J. Intell. Mater. Syst. Struct. 13, 401-404 (2002).

Kuzhir, P., M. T. López-López, G. Vertelov, C. Pradille and G. Bossis, "Shear and squeeze rheometry of suspensions of magnetic polymerized chains," Rheol. Acta (2007), in press. DOI $10.1007 / \mathrm{s} 00397-007-0230-7$ 
Larson, R. G., The Structure and Rheology of Complex Fluids. Oxford University Press, New York, 1999.

Liu, J., G. A. Flores and R. Sheng, "In-vitro investigation of blood imbolization in cancer treatment using magnetorheological fluids,” J. Magn. Magn. Mater. 225, 209-217 (2001).

López-López, M. T., P. Kuzhir, S. Lacis, G. Bossis, F. Gonzalez-Caballero and J. D. G. Duran, "Magnetorheology for suspensions of solid particles dispersed in ferrofluids," J. Phys.Condens. Matter 18, S2803-S2813 (2006a).

López-López, M. T., A. Zugaldía, F. González-Caballero and J. D. G. Durán, "Sedimentation and redispersion phenomena in iron-based magnetorheological fluids," J. Rheol. 50, 543-560 (2006b).

López-López, M. T., G. Vertelov, P. Kuzhir, G. Bossis and J. D. G. Duran, "New magnetorheological fluids based on magnetic fibers," J. Mater. Chem. 17, 3839-3844 (2007).

Mason, S. G., "The flocculation of pulp suspensions and the formation of paper," TAPPI J. 33, 440-444 (1950).

Ngatu, G. T., N. M. Wereley, J. O. Karli and R. C. Bell, "Dimorphic Magnetorheological Fluids: Exploiting Partial Substitution of Microspheres by Nanowires," Smart Mater. Struct. 17, 045022 (2008). 
Ngatu, G. T. and N. M. Wereley, "Viscometric and sedimentation characterization of bidisperse magnetorheological fluids," IEEE Trans. Magn. 43, 2474-2476 (2007).

Pu, H. T., F. J. Jiang, Z. L. Yang, B. Yan and X. Liao, "Effects of polyvinylpyrrolidone and carbon nanotubes on magnetorheological properties of iron-based magnetorheological fluids," J. Appl. Polym. Sci. 102, 1653-1657 (2006).

Rankin, P. J., J. M. Ginder and D. J. Klingenberg, “Electro- and magneto-rheology,” Curr. Opin. Colloid Interface Sci. 3, 373-381 (1998).

Schmid C. F., Switzer L.H., Klingenberg D.J., "Simulations of fiber flocculation: Effects of fiber properties and interfiber friction”, J. Rheol. 44, 781-809 (2000).

See, H., "Magneto-rheological suspensions - Physical mechanisms and modeling," Nihon Reoroji Gakkaishi 31, 297-303 (2003).

Sheng, R., G. A. Flores and J. Liu, "In vitro investigation of a novel cancer therapeutic method using embolizing properties of magnetorheological fluids” J. Magn. Magn. Mater., 194, 167-175 (1999).

Switzer, L. H. and D. J. Klingenberg, "Flocculation in simulations of sheared fiber suspensions," Int. J. Multiph. Flow 30, 67-87 (2004).

Toll, S. and J.-A. E. Manson, "Dynamics of a planar concentrated fiber suspension with nonhydrodynamic interaction,” J. Rheol. 38, 985-997 (1994). 
Viota, J. L., F. Gonzalez-Caballero, J. D. G. Duran and A. V. Delgado, "Study of the colloidal stability of concentrated bimodal magnetic fluids", J. Colloid Interface Sci., 309, 135-139 (2007).

Volkova, O., "Study of rheology of suspensions of magnetic particles," Ph.D. Thesis, Université de Nice-Sophia Antipolis, 1998.

Volkova, O., G. Bossis, M. Guyot, V. Bashtovoi and A. Reks, "Magnetorheology of magnetic holes compared to magnetic particles,” J. Rheol. 44, 91-104 (2000).

Wereley, N. M., A. Chaudhuri, J. H. Yoo, S. John, S. Kotha, A. Suggs, R. Radhakrishnan, B. J. Love and T. S. Sudarshan, "Bidisperse magnetorheological fluids using Fe particles at nanometer and micron scale,” J. Intell. Mater. Syst. Struct. 17, 393-401 (2006).

Wilhelm, C., F . Gazeau and J.-C. Bacri, "Magnetic micromanipulation in the living cell" Europhys. News, 3, 89-92 (2005). 


\section{FIGURE CAPTIONS}

Figure 1. SEM image of the cobalt fibers.

Figure 2. Magnetization, $M$, as a function of magnetic field strength, $H$, for dry cobalt powders at $20^{\circ} \mathrm{C}$. O: cobalt fibers; $\mathbf{\square}$ : Cobalt spheres. Solid lines corresponds to the FröhlichKennelly fitting (equation 1).

Figure 3. Schema of the non-commercial electromagnet used in the magnetorheological experiments at medium and high fields.

Figure 4. Radial distribution of the magnetic flux density in the measuring gap calculated by means of finite element method. The radius of the upper plate of the rheometer is $10 \mathrm{~mm}$.

Figure 5. Shear stress as a function of shear rate for a suspension containing $5 \mathrm{vol} . \%$ of cobalt fibers. The values of the applied magnetic field strength are indicated.

Figure 6. Shear stress plotted as a function of shear rate for suspensions containing 3 vol. $\%$ of cobalt spheres ( $\square$ ) or fibers $(\circ)$ in silicone oil, under the presence of an applied magnetic field strength of $30.55 \mathrm{kA} \mathrm{m}^{-1}$. (a) Linear data acquisition; (b) logarithmic data acquisition.

Figure 7. Static yield stress plotted as a function of the magnetic field strength for suspensions of cobalt particles in silicone oil. Volume fraction of solids: $\mathbf{m}: 1 \% ; \circ: 3 \%$; $5 \% ; \nabla: 7 \%$. (a) suspensions of cobalt spheres; (b) suspensions of cobalt fibers. 
Figure 8. Dynamic yield stress plotted as a function of the magnetic field strength for suspensions of cobalt particles in silicone oil. Volume fraction of solids: $\mathbf{m}: 1 \% ; 0: 3 \% ; \boldsymbol{\Delta}$ : $5 \% ; \nabla: 7 \%$. (a) suspensions of cobalt spheres; (b) suspensions of cobalt fibers.

Figure 9. (a-e) photos of planar structures of diluted suspensions of cobalt fibers (solid concentration 0.1 vol.\%) confined between two parallel glass slides (the gap was fixed to 0.15 $\mathrm{mm}$ ). (a) in the absence of applied magnetic field; (b-c) in the presence of an applied magnetic field parallel to the glass slides; (d-e) in the presence of an applied magnetic field normal to the glass slides: unstrained suspension (d) and strained suspension (e). (f) photo of a 3D structure of a model fiber suspension under the presence of applied magnetic field. 


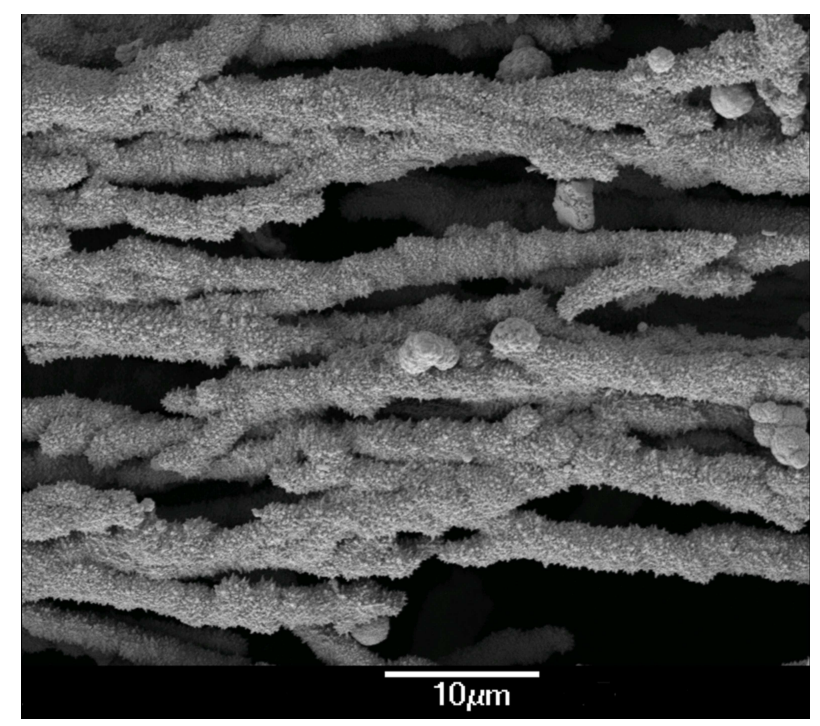

Fig.1. 


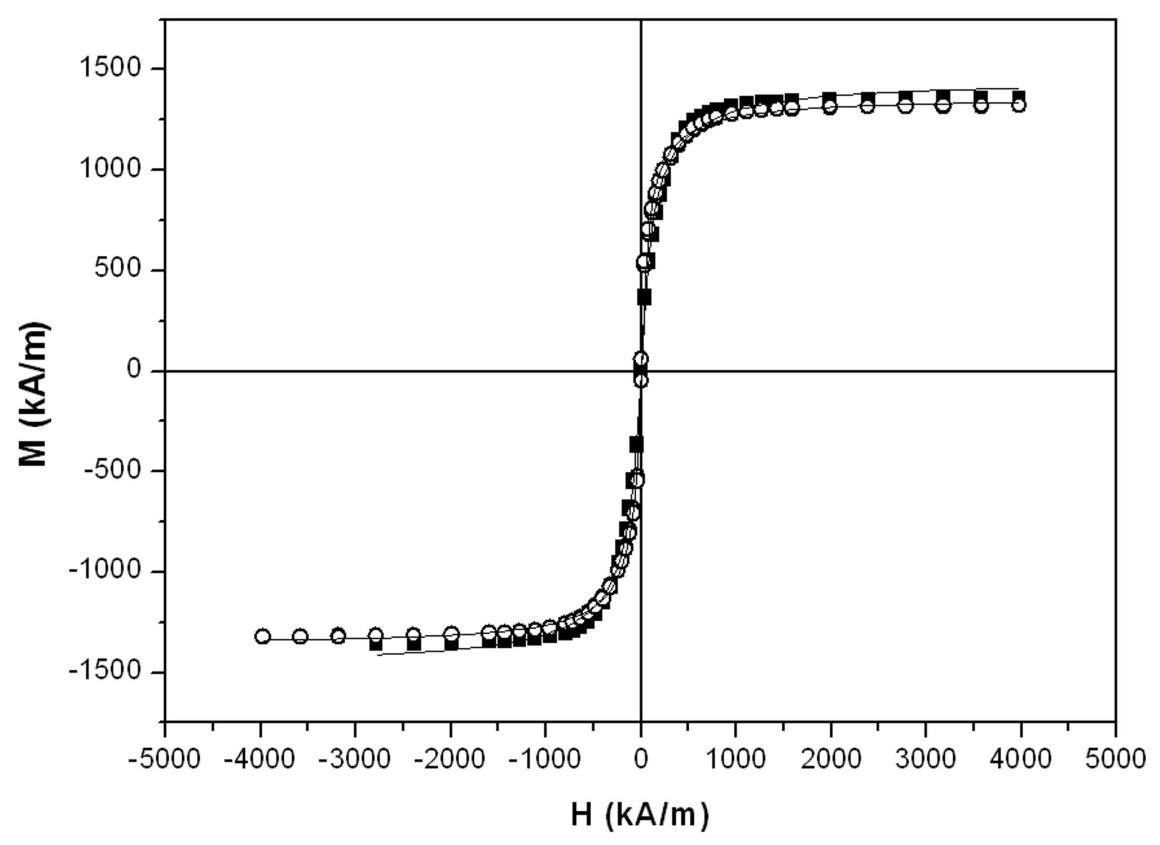

Fig.2. 


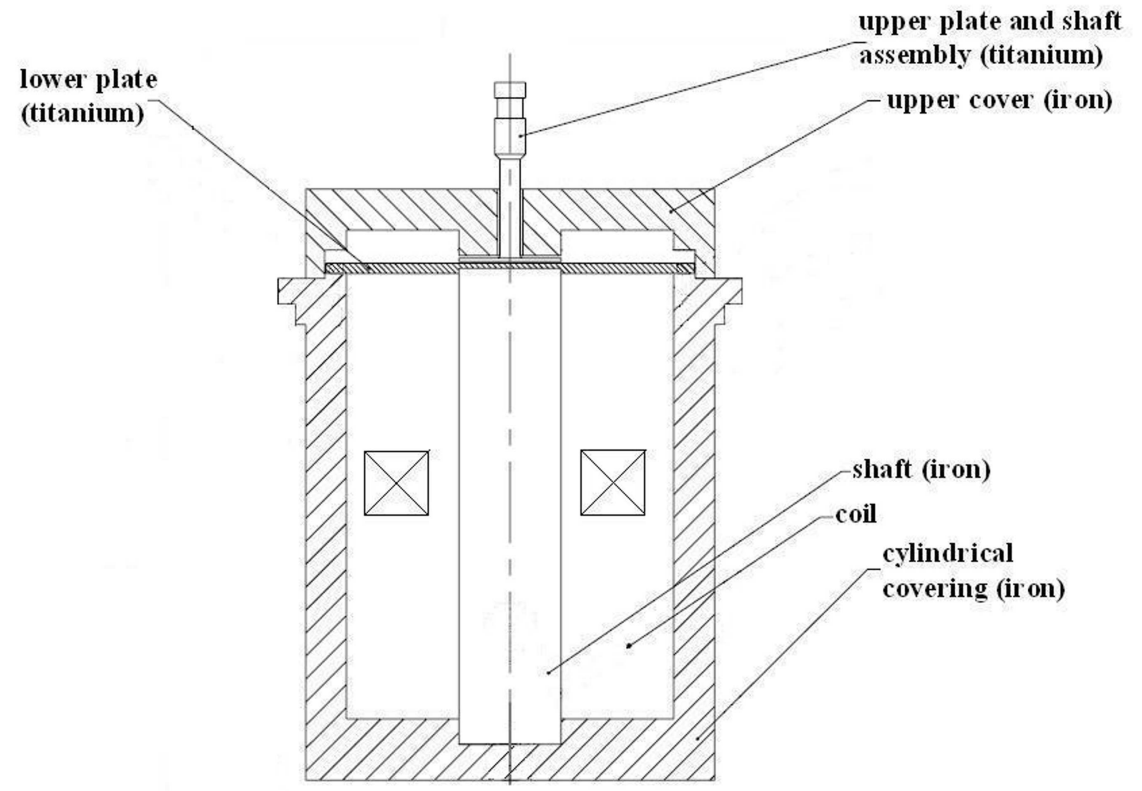

Fig. 3 


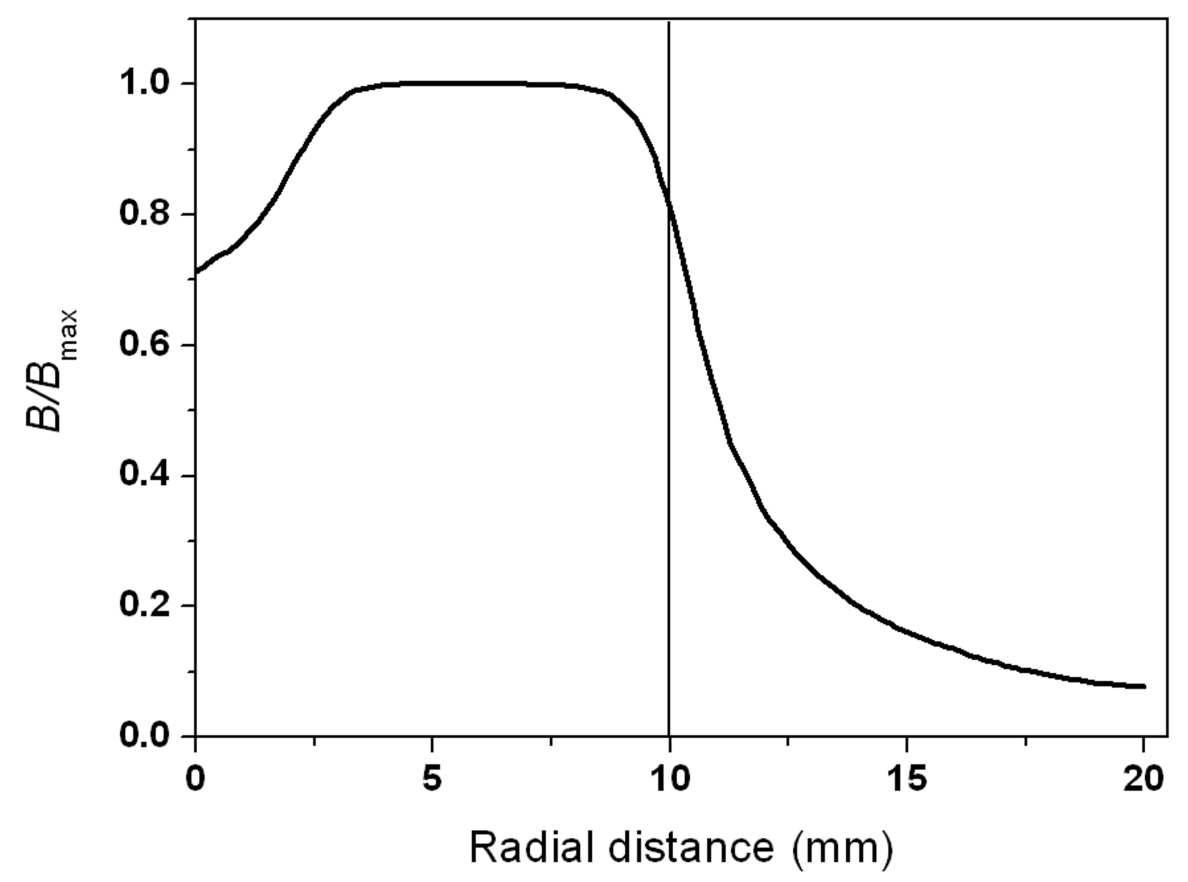

Fig. 4 


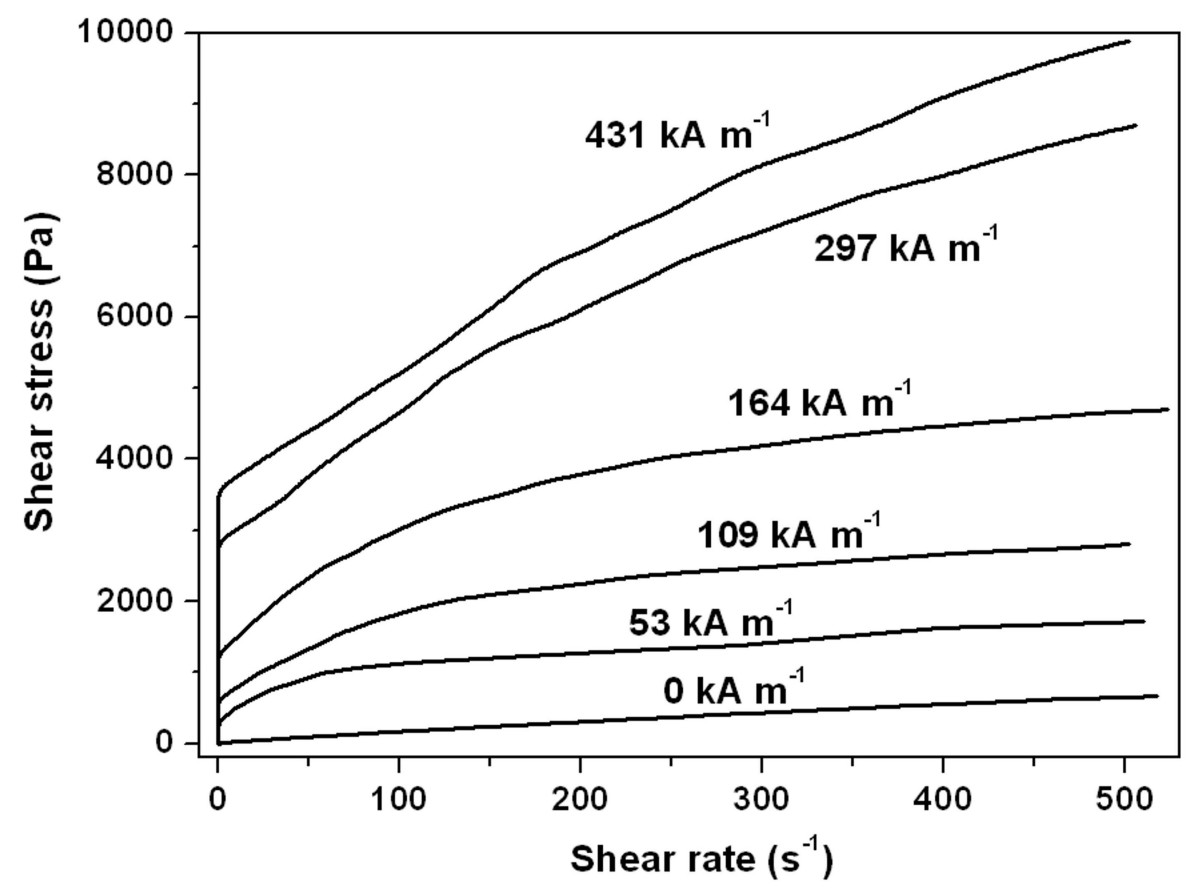

Fig.5. 

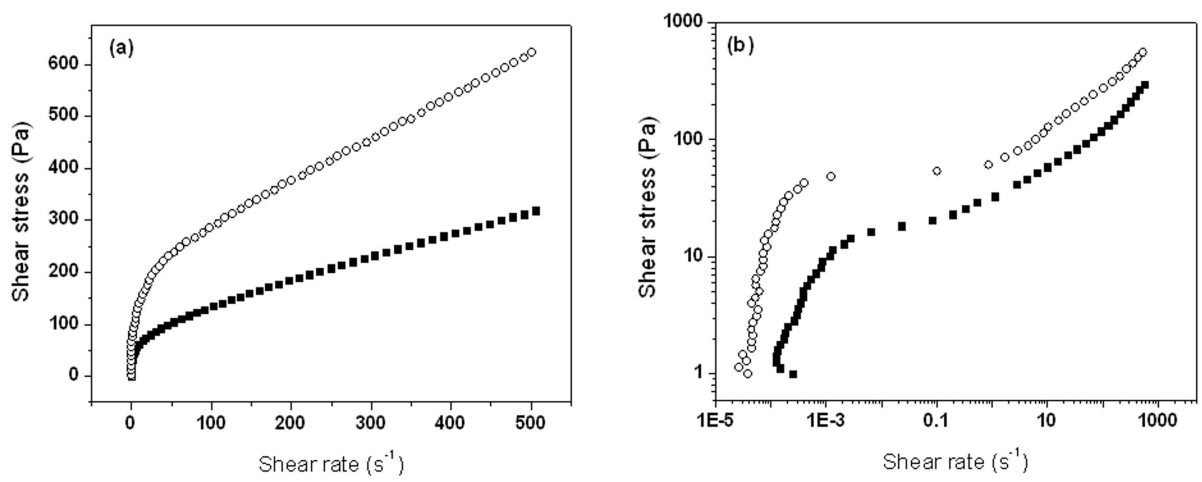

Fig.6 

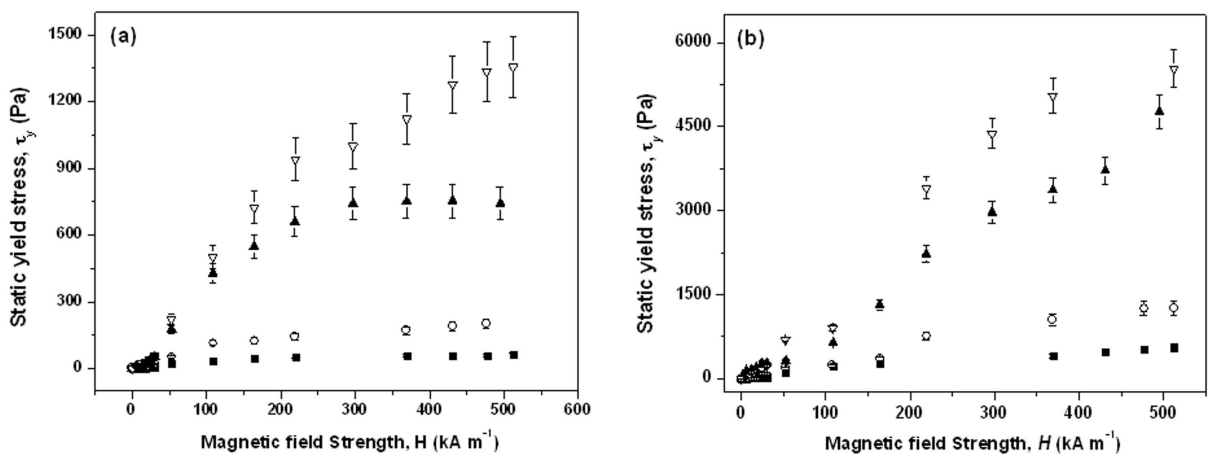

Fig.7. 

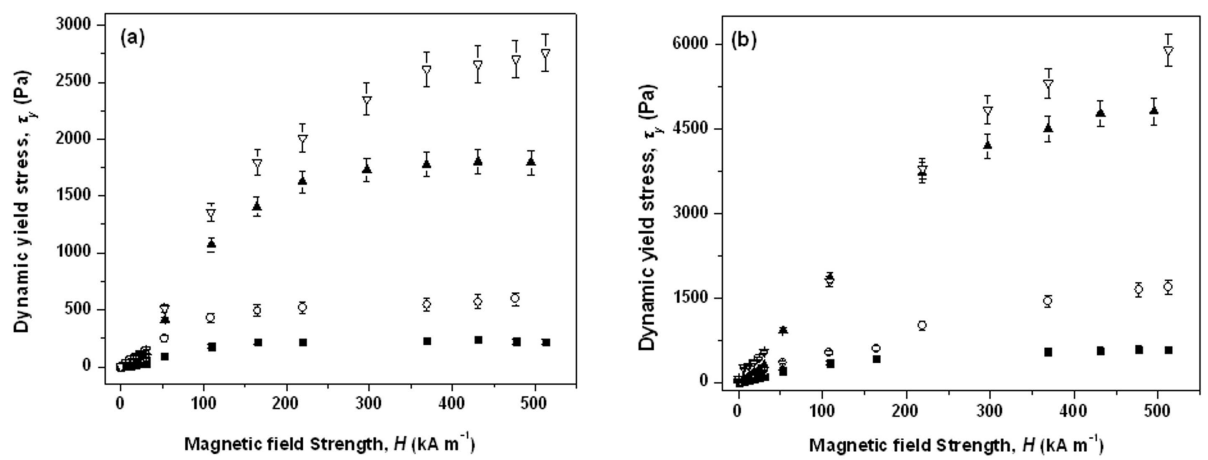

Fig.8. 
(a) $H=0$

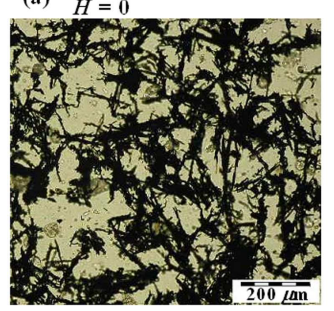

(d) $\odot H=33 \mathrm{kA} \mathrm{m}^{-1}$

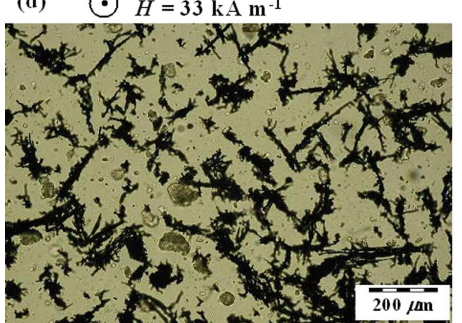

(b) $H=25 \mathrm{kA} \mathrm{m}^{-1}$

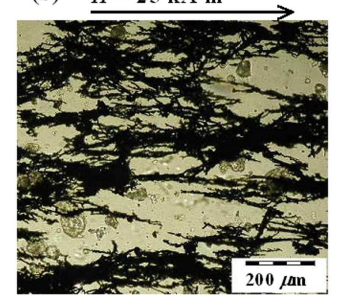

(e) $\odot H=33 \mathrm{kA} \mathrm{m}^{-1}$

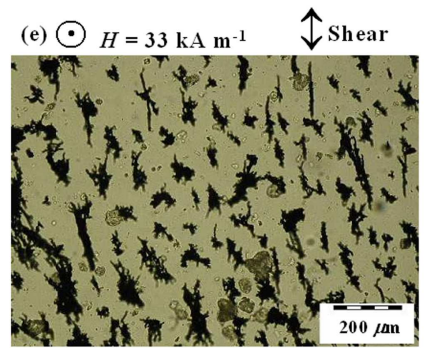

Fig.9. (c) $\underline{H=25 \mathrm{kA} \mathrm{m}^{-1}}$

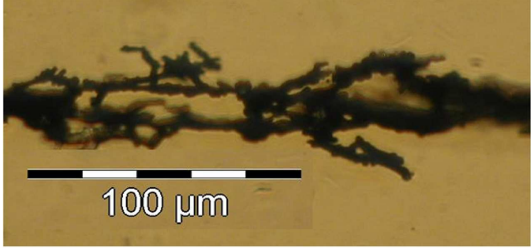

(f) $\uparrow_{H=32 \mathrm{kA} \mathrm{m}^{-1}}$

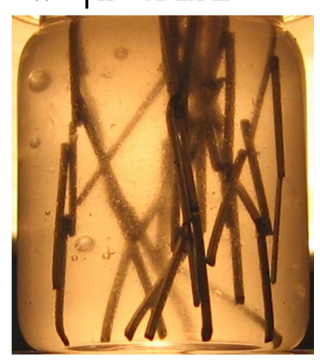

